\title{
The comprehensive complication index (CCI) is a more sensitive complication index than the conventional Clavien-Dindo classification in radical gastric cancer surgery
}

\author{
Tae-Han Kim ${ }^{1} \cdot$ Yun-Suhk Suh ${ }^{1} \cdot$ Yeon-Ju Huh $^{1} \cdot$ Young-Gil Son $^{1} \cdot$ \\ Ji-Ho Park ${ }^{1}$ - Jun-Young Yang ${ }^{1}$ - Seong-Ho Kong ${ }^{1}$ - Hye Seong Ahn ${ }^{3}$. \\ Hyuk-Joon Lee ${ }^{1,2} \cdot$ Ksenija Slankamenac $^{4} \cdot$ Pierre Alain Clavien ${ }^{4} \cdot$ \\ Han-Kwang Yang ${ }^{1,2}$
}

Received: 22 January 2017/Accepted: 18 May 2017/Published online: 8 June 2017

(C) The International Gastric Cancer Association and The Japanese Gastric Cancer Association 2017

\begin{abstract}
Background The comprehensive complication index (CCI) integrates all complications of the Clavien-Dindo classification (CDC) and offers a metric approach to measure morbidity. The aim of this study was to evaluate the CCI at a high-volume center for gastric cancer surgery and to compare the CCI to the conventional CDC.

Methods Clinical factors were collected from the prospective complication data of gastric cancer patients who underwent radical gastrectomy at Seoul National University Hospital from 2013 to 2014. CDC and CCI were calculated, and risk factors were investigated. Correlations and generalized linear models of hospital stay were compared between the CCI and CDC. The complication monitoring model with cumulative sum control-CCI (CUSUMCCI) was displayed for individual surgeons, for comparisons between surgeons, and for the institution.

Results From 1660 patients, 583 complications in 424 patients $(25.5 \%)$ were identified. The rate of CDC grade IIIa or greater was $9.7 \%$, and the overall CCI was
\end{abstract}

Electronic supplementary material The online version of this article (doi:10.1007/s10120-017-0728-3) contains supplementary material, which is available to authorized users.

Han-Kwang Yang

hkyang@snu.ac.kr

1 Department of Surgery, Seoul National University College of Medicine, Seoul, Korea

2 Cancer Research Institute, Seoul National University College of Medicine, Seoul, Korea

3 Department of Surgery, Seoul National University-SMG Boramae Medical Center, Seoul, Korea

4 Department of Surgery, University Hospital Zurich, Zurich, Switzerland
$5.8 \pm 11.7$. Age, gender, Charlson score, combined resection, open method, and total gastrectomy were associated with increased CCI $(p<0.05)$. The CCI demonstrated a stronger relationship with hospital stay $(\rho=0.721, p<0.001)$ than did the CDC $(\rho=0.634$, $p<0.001$ ). For prolonged hospital stays ( $\geq 30$ days), only the CCI showed a moderate correlation $(\rho=0.544$, $p=0.024)$, although the CDC did not. The CUSUM-CCI model displayed dynamic time-event differences in individual and comparison monitoring models. In the institution monitoring model, a gradual decrease in the CCI was observed.

Conclusions The CCI is more strongly correlated with postoperative hospital stay than is the conventional CDC. The CUSUM-CCI model can be used for the continuous monitoring of surgical quality.

Keywords Morbidity $\cdot$ Stomach $\cdot$ Neoplasm

\section{Introduction}

Surgery is the mainstay of treatment for patients with gastric cancer. Despite the best efforts of surgeons, the incidence of complications following gastrectomy is reported to range from $15 \%$ to $25 \%$ [1-4]. As surgical techniques are improving and the focus of the surgeons shifts from oncological outcomes to the quality of life and postoperative safety of patients, control and monitoring of complications are necessary.

Postoperative complications are graded by the ClavienDindo classification (CDC) according to the degree of invasiveness required by the treatment response [5, 6]. Because of the reproducible interpretation of complications, the CDC has been widely applied in many fields of 
surgery [7-9]. In our center, we have reported the CDC in gastrectomy patients using the prospective complication database [3]. However, patients who suffer severe complications that involve organ failure usually experience multiple collateral complications [10]. Because CDC grading is based on only the most severe forms of complications and ignores other minor complications [9], the comprehensive complication index (CCI) was recently introduced and validated in a large cohort of patients who underwent abdominal surgery for various reasons [11]. A key feature of the CCI is the mathematical summation of the complications graded as in the conventional CDC, which is displayed as a continuous figure from 0 (no complications) to 100 (death), regardless of the number and severity of the complication(s) [12].

Because the CCI measures the overall magnitude of all complications, continuous monitoring of the CCI can mirror surgical performance and provide feedback to the surgeon. The continuous time-sequenced recording of the CCI can provide a reference to monitor the surgical performance of a group of surgeons.

The aim of this study was to evaluate the CCI and conventional CDC in gastrectomy patients at a high-volume gastric cancer surgery center and apply this evaluation to monitor complications in a time sequence.

\section{Methods}

\section{Patient data}

Prospective data from patients who underwent gastrectomy for reasons of gastric adenocarcinoma at Seoul National University Hospital from 2013 to 2014 were included in the study. Advanced gastric cancer patients were treated with conventional laparotomy, and laparoscopic procedures were performed in patients with clinically early gastric cancer. The technical details are described in a previous publication [3].

Data on age, gender, body mass index (BMI), operation method (open, laparoscopy, or robot), operation type (distal gastrectomy, total gastrectomy, or pylorus-preserving gastrectomy), extent of lymph node dissection (less than D2 or D2), number of retrieved lymph nodes, combined resection, pathological stage (7th AJCC TNM classification), and record of postoperative hospital stay and visits were collected [13, 14].

Comorbidity data were collected and graded using the Charlson comorbidity index, which is the most widely used method to quantify the overall burden of comorbidities and includes 19 medical conditions with corresponding weights [15]. The index has been investigated as an effective predictor of health outcomes in patients with gastric cancer who have undergone surgical resection. Previous reports show higher Charlson comorbidity indexes are associated with poorer short-term surgical outcome after gastric cancer surgery [16].

\section{Complication data}

A prospective database collection from 2013 to 2014 was used. In this period, daily clinical courses and events were prospectively described by the attending surgeons, and all complications were converted to the CDC in weekly meetings; all team faculty members attended these meetings to form agreements for grading with a unified application to all cases. Complications that occurred within 30 postoperative days were considered relevant to the surgical procedure regardless of patient re-admission or re-visit. Complications that occurred after 30 postoperative days were collected when they occurred during the same hospitalization period.

\section{Calculation for the CCI}

The CCI formula, which integrates every postoperative complication of a patient, is based on methods from operations risk index analysis used in economic science. Each Clavien-Dindo complication grade has an attributed severity weight, which was calculated from the multiplication of the physicians' and patients' perspective of harm. The square root of the sum of all weights amounts to the CCI after dividing by 2 .

$\mathrm{CCI}=\sqrt[2]{\sum \mathrm{MRV} \text { phys } \times \mathrm{MRV} \text { pat }} / 2$,

where $M R V$ phys is the median reference value of physicians and $M R V$ pat is the median reference value of patients.

For feasible applications, it can be easily assessed by the CCI calculator available at website (http://www.assessur gery.com). From our data, integrated complication data for each patient were entered into the CCI calculator, and CCI values were computed and summed for analysis $[12,17]$.

\section{Criteria for patient discharge}

In our routine practice, serum blood tests are performed to screen any postoperative complications. Laboratory tests, including complete blood count, electrolytes, admission panel [i.e., calcium, phosphorus, glucose, blood urea nitrogen (BUN), uric acid, cholesterol, protein, albumin, total bilirubin, alkaline phosphatase, aspartate aminotransferase, alanine aminotransferase, and creatinine], and C-reactive protein (CRP) level, were measured on postoperative days (POD) 2, 5, and 7. If the patient could ingest 
water successfully, a semi-fluid diet was allowed on PODs 4 and 5 followed by a semi-blended diet on POD 6 and 7 . In the absence of complications or complaints after ingesting the semi-blended diet, patients were considered capable of being discharged. If at any point a patient developed fever $\left(\geq 37.8^{\circ} \mathrm{C}\right)$, an extensive search for its cause was engaged and discharge was postponed.

\section{Continuous complication monitoring chart for surgical quality improvement}

The cumulative sum control (CUSUM) technique is a graphical method that detects data trends to monitor outcomes in surgical procedures [18]. This method reveals the accumulated difference between the event and target value at each observation time point. Logically, the cumulative sum is $\sum_{i=1}^{n}(\mathrm{Xi}-\mu)$, where $\mathrm{Xi}$ is the CCI of each operation, and $\mu$ is the target value. The CUSUM score graph shows a steady decrease when no complication event is noted, whereas the graph shows a sharp increase when complications with high CCI occur. In our study, three scenarios were tested with the CCI-CUSUM model. First, the CCI of individual surgeons were shown in the CUSUM chart with accumulated complication rates. The target value was set for the average CCI of the year 2013, and the case-event CUSUM chart for 2014 was shown for two surgeons, A and B. Second, to compare the surgical outcomes between surgeons, the target was set for the average CCI for both surgeons in 2013, and the combined CUSUM chart for cases in 2014 was presented. Finally, overall monitoring of surgical complications at the institution for 2014 was depicted with a target value of the average institutional CCI in 2013.

\section{Statistical analysis}

To compare the CCI and the CDC grading system, two methods were tested. First, correlation analysis was performed with Spearman's rank test. The correlation coefficient score of the CCI with postoperative hospital stay was compared to the score of CDC.

Univariate analysis was performed by $\chi^{2}$ test, $t$ test, and analysis of covariance (ANCOVA) to compare the difference between CCI, CDC (higher than grade II), and complication rate (of any grade) in various factors. From this analysis, factors with $p$ value of $<0.05$ were assembled for multivariate analysis with each complication system. The multivariate analysis was run by regression model, and because the $R^{2}$ value of each model represents the "proportion of the explained data" from the total data set, the $R^{2}$ value of the final model from each complication system was compared between the CCI model and the CDC model [19].
Statistical Package for Social Science version 21.0 (SPSS, Chicago, IL, USA) was used in all analyses. All $p$ values are two sided, and $p$ values $<0.05$ were considered statistically significant.

\section{Results}

\section{Patients and complications}

A total of 1660 patients were evaluated. The patient characteristics, described in Table 1, included a mean age of $60.5 \pm 11.8$ years, $64 \%$ male predominance, and a BMI of $22.8 \pm 3.2\left(\mathrm{~kg} / \mathrm{m}^{2}\right)$. The mean postoperative hospital stay was $10.4 \pm 7.6$ days: $8.05 \pm 2.2$ days in the patient group without complications and $17.8 \pm 13.5$ days in the complicated patient group with complications. Among the total of 1660 patients, 424 patients $(25.5 \%)$ developed complications; $71 \%$ (304 of 424) of the complicated patients presented with a single complication, and $29 \%$ of these patients presented with multiple complications. Complications following re-operation were integrated into the primary operation.

The details of postoperative complications in the Clavien-Dindo classification are $7.8 \%$ for grade I, $13.9 \%$ for grade II, $10.8 \%$ for grade III, and $1.1 \%$ for grade IV (supplementary data). The rate of severe complication ( $\geq$ CDC grade III) was $11.9 \%$. One patient experienced a grade $\mathrm{V}$ complication from an acute myocardial infarction following the operation. Pulmonary complications (6.8\%) were the most frequent, followed by fluid collection (5.2\%) and wound complications (4.3\%). Eighteen patients (1.0\%) underwent re-operation; 9 developed wound dehiscence, 2 intraabdominal bleeding, 2 anastomosis stenosis, 1 delayed intestinal activity, 1 anastomotic leakage, 1 vascular complication, and 1 laryngo-microscopic surgery for vocal hoarseness following the primary operation. One patient underwent a laparotomy for a diaphragmatic hernia that developed following total gastrectomy.

In patients who experienced complications, the mean CCI was $23.6 \pm 9.7$ (median 22.6); 133 patients with a single complication of CDC grade, 87 patients with a single complication of CDC grade IIIa, and 66 patients with a single complication of CDC grade I showed a CCI of 8.7.

The distribution of complicated patients according to $\mathrm{CCI}$ is shown in Fig. 1. Because each complication grade is designated to prefixed scores (grade $\mathrm{I}=8.7$, grade $\mathrm{II}=20.9$, grade $\mathrm{IIIa}=26.2$, grade $\mathrm{IIIb}=33.7$, grade $\mathrm{IVa}=42.4$, grade $\mathrm{IVb}=46.2$ ) and the majority of grade I and grade II complicated patients show a single complication, a spike for CCI of 8.7 and 20.9 can be observed. Patients featuring the highest complication of grade III show a wide CCI distribution of CCI between 26.2 and 40.5, depending 
Table 1 Patient overview $(n=1660)$

\begin{tabular}{|c|c|c|}
\hline Variables & Mean $\pm \mathrm{SD}$ & Number $(\%)$ \\
\hline Age (years) & $60.5 \pm 1.8$ & \\
\hline Sex ratio (male:female) & $1.8: 1$ & \\
\hline BMI $\left(\mathrm{kg} / \mathrm{m}^{2}\right)$ & $22.8 \pm 3.2$ & \\
\hline \multicolumn{3}{|l|}{ Charlson Score } \\
\hline 2 & & $1131(68.1)$ \\
\hline 3 & & $342(20.5)$ \\
\hline$\geq 4$ & & $187(11.4)$ \\
\hline \multicolumn{3}{|l|}{ Stage (7th AJCC) } \\
\hline I & & $1108(67.0)$ \\
\hline II & & $232(13.9)$ \\
\hline III & & $274(16.4)$ \\
\hline IV & & $46(2.8)$ \\
\hline \multicolumn{3}{|l|}{ Operation type } \\
\hline PPG & & $358(21.6)$ \\
\hline DG & & $912(54.9)$ \\
\hline TG & & $390(23.5)$ \\
\hline \multicolumn{3}{|l|}{ Operation method } \\
\hline Laparoscopy & & $998(60.1)$ \\
\hline Robot & & $85(5.1)$ \\
\hline Open & & $577(34.8)$ \\
\hline \multicolumn{3}{|l|}{ Combined resection } \\
\hline None & & $1,380(83.1)$ \\
\hline Resected & & $280(16.9)$ \\
\hline \multicolumn{3}{|c|}{ Extent of lymph node dissection } \\
\hline Under D2 & & $928(56)$ \\
\hline D2 & & $732(44)$ \\
\hline Patients with complications & & $424(25.5)$ \\
\hline \multicolumn{3}{|l|}{ Number of complications } \\
\hline 1 & & $304(71.6)$ \\
\hline 2 & & $94(22.1)$ \\
\hline 3 & & $20(4.7)$ \\
\hline 4 & & $5(1.1)$ \\
\hline 5 & & $1(0.2)$ \\
\hline 6 & & $1(0.2)$ \\
\hline Hospital days stay overall & $10.5 \pm 8.2$ & \\
\hline Without complication & $8.1 \pm 2.2$ & \\
\hline With complication & $17.8 \pm 13.5$ & \\
\hline
\end{tabular}

$P P G$ pylorus-preserving gastrectomy, $D G$ distal gastrectomy, $T G$ total gastrectomy,

on the amount and severity of the other complications. In patients with the highest complication of grade IV, a wide distribution of CCI (42.4-72.9) also was seen.

The surgical and clinical factors relevant to complications are shown in Table 2. The CCI was significantly different, depending on gender, age, Charlson score, stage, operation type, approach method, combined resection, and extent of lymph node dissection via univariate analysis. In contrast, age group and Charlson score were not associated with differences in the CDC.

Significant factors were collected and tested in the ageand BMI-adjusted model and were assembled in a linear model for CCI. Age, gender, Charlson score, combined resection, approach method, and operation type were associated with increased CCI $(p<0.05)$ (Table 3).

\section{Relationship with hospital stay}

Figure $2 \mathrm{a}, \mathrm{b}$ shows the mean hospital stay according to the highest grade of the patient CDC and CCI. For the CDC, from grade I to III, each escalation in the CDC grade was associated with an increase in the duration of the hospital stay $(p<0.05)$, whereas a decrease in hospital stay was observed for patients with stage IV complications $(p=0.891)$. In contrast, the CCI continually increased with the increase in hospital stay for each subgroup, and there was a marked increase in hospital stay in the CCI 25.1-35 group. A total of 1574 (94.7\%) cases were collected to evaluate correlations. The CCI exhibited a strong positive relationship $(\rho=0.724, p<0.001)$ compared to the moderate correlation of the CDC $(\rho=0.636$, $p<0.001)$. In patients with a prolonged hospital stay ( $>30$ days), the CCI displayed a moderate correlation of $\rho=0.544(p=0.024)$; however, the CDC was not associated with a significant correlation at $\rho=0.405$ $(p=0.107)$.

\section{Multivariate analysis}

Patient factors (age, gender, BMI, stage, and Charlson score), surgical factors (operation type, approach method, combined resection, and extent of dissection) and CCI or CDC were entered for the hospital stay-related general linear model. Each variable was sequentially studied for an interaction with the CCI or CDC.

The significant variables entered for the final modeling other than the complication scales were the Charlson score and operation type (Table 4). $R^{2}$ was 0.505 for the CCI model and 0.403 for the CDC model in the multivariate analysis of overall hospital stay. $R^{2}$ values for the CCI and CDC models were 0.387 and 0.297 , respectively, in the analysis of a prolonged hospital stay.

\section{Monitoring for the complication rates of individual surgeons}

To monitor each surgeon's complication outcome in the year 2014, the target goal value of CCI in each surgeon was set for the mean value of year 2013 (Fig. 3a). For surgeon $\mathrm{A}$, target value was set at CCI of 5.4 and the CCI-CUSUM chart of 356 cases of year 2014 was depicted. In this figure, 


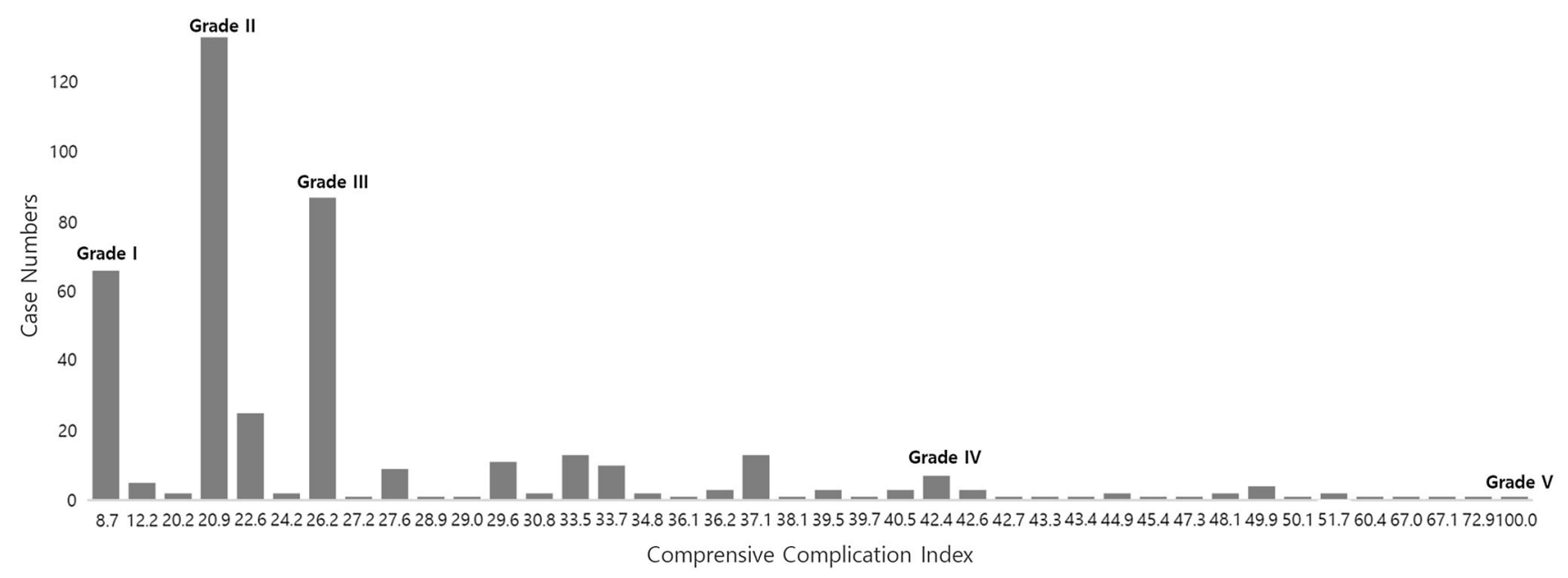

Fig. 1 Overview of comprehensive complication index (CCI) in gastrectomy patients

the CUSUM score shows irregular fluctuation during the observation period, shows the highest peak at case 242, and draws a gradual decrease, resulting in the CCI score of -53.7 in the last case, which indicates improvement in the CCI. For surgeon B, the target value of 7.4 was applied in 302 cases performed in 2014. In Fig. 3b, the CUSUM score shows gradual decrease until case 103 and shows notable increase from case 190 to case 200 . The result CCI for surgeon B was -185.2 , which indicates improved complication outcome compared to 2013.

To compare the surgical outcome of the two surgeons evaluated, the target value was set in the mean CCI score for both surgeons A and B in the year 2013. The CUSUMCCI chart was depicted for each surgeon with this target value. Figure $3 \mathrm{~b}$ shows the CUSUM score for the comparison of the two surgeons. According to this chart, although surgeon B exhibited a steady increase throughout the period, surgeon A exhibited a gradual decrease in CUSUM score.

The CUSUM chart for the institutional CCI revealed a dynamic change throughout the observed period, as shown in Fig. 3c, and the trend line of institutional CCI, $y=-0.0978 x-0.5802$, demonstrated a gradual decrease in time sequence.

\section{Discussion}

In this study, the CCI system was validated in a highvolume center where a prospectively collected complication database was available from a homogeneous, wellcontrolled surgical practice. This study demonstrates the superiority of the CCI over the conventional CDC system in patients who have undergone gastrectomy for gastric cancer. An evaluation of the overall postoperative complications in a patient should consider not only the number but also the severity of complications. This study shows that grading systems that use only the most severe complications, not including the collateral complications or total number of complications (CDC), produce an insufficient report compared to the CCI. As the CCI system is a continuous scoring variable, it can be easily combined to form the CUSUM-CCI model, providing charts to monitor individual CCI and compare the performances of various surgeons as well as to monitor institutional surgical outcomes.

To date, complication reports based on the CCI system are quite limited. In recent prospective studies, the CCI values for NOTES cholecystectomy (mean, $3.3 \pm 6.3$ ) and conventional open esophageal resection (median, 12) have been reported [20, 21]. According to these previous reports and our current report of the CCI for laparoscopic gastrectomy (mean, $4.9 \pm 9.5$ ) and conventional open gastrectomy (mean, $9.0 \pm 13.4$ ), the CCI values appear to be good estimates for surgical trauma in each procedure.

Complications arise from surgical stress, or especially from age and physical frailty, which have recently been regarded as predictive factors for increased morbidity $[22,23]$. To take these factors into consideration, we applied the well-established Charlson score in our study [4, 24, 25]. The CCI was increased in patients with higher Charlson scores but the CDC was not. In all multivariate analyses, the Charlson score was regarded as a significant predisposing factor for both CCI and hospital stay when associated with the CCI. Although the CDC score showed incremental tendency along with the score, it failed to display statistical significance in this analysis, showing poorer correlation compared to the CCI system. This observation does not reflect the failure of CDC classification but rather shows the nature of complication in comorbid patients. Our data show 
Table 2 Clinical factors and complications $(n=1660)$

\begin{tabular}{|c|c|c|c|c|c|c|c|}
\hline Variables & Numbers $(\%)$ & Complication rate $(\%)$ & $p$ value & $\mathrm{CDC} \geq \mathrm{Gr}$ III $(\%)$ & $p$ value & Mean CCI (SD) & $p$ value \\
\hline Overall & 424 & 25.5 & & 97 & & $5.8(11.7)$ & \\
\hline \multicolumn{5}{|c|}{ Within complication group } & & $23.6(9.7)$ & \\
\hline \multicolumn{8}{|l|}{ Sex } \\
\hline Male & $1065(64.2)$ & 29.0 & & 10.4 & & $6.7(12.4)$ & \\
\hline Female & $595(35.8)$ & 18.9 & $<0.001$ & 7.1 & 0.025 & $4.1(9.9)$ & $<0.001$ \\
\hline \multicolumn{8}{|l|}{ Age } \\
\hline-40 & $98(5.9)$ & 22.4 & & 6.7 & & $4.2(8.8)$ & \\
\hline $41-50$ & $242(14.6)$ & 19.0 & & 7.5 & & $4.3(8.2)$ & \\
\hline $51-60$ & 475 (28.6) & 22.4 & & 9.1 & & $5.3(10.4)$ & \\
\hline $61-70$ & $448(27.0)$ & 27.1 & & 9.1 & & $6.6(12.0)$ & \\
\hline $71-$ & 397 (23.9) & 31.4 & 0.003 & 10.7 & 0.541 & $8.3(11.9)$ & $<0.001$ \\
\hline \multicolumn{8}{|l|}{ BMI $\left(\mathrm{kg} / \mathrm{m}^{2}\right)$} \\
\hline-18.5 & $117(7)$ & 29.8 & & 10.9 & & $6.8(12.5)$ & \\
\hline $18.5-25$ & 1145 (69) & 25.4 & & 9.2 & & $5.9(11.5)$ & \\
\hline $25-$ & $398(24)$ & 23.8 & 0.380 & 8.8 & 0.777 & $5.4(10.1)$ & 0.744 \\
\hline \multicolumn{8}{|l|}{ Charlson Score } \\
\hline 2 & $1131(68.1)$ & 21.5 & & 8.8 & & $5.3(10.7)$ & \\
\hline 3 & $342(20.5)$ & 27.4 & & 10.2 & & $6.7(12.9)$ & \\
\hline$\geq 4$ & $187(11.4)$ & 28.9 & 0.012 & 10.2 & 0.644 & $9.9(15.2)$ & $<0.001$ \\
\hline \multicolumn{8}{|c|}{ Stage (7th AJCC) } \\
\hline I & 1108 (66.7) & 19.9 & & 7.5 & & $5.0(10.7)$ & \\
\hline II & $232(14.0)$ & 25.8 & & 9.4 & & $6.5(11.8)$ & \\
\hline III & $274(16.5)$ & 35.8 & & 15.7 & & $8.7(12.0)$ & \\
\hline IV & $46(2.8)$ & 32.6 & $<0.001$ & 15.2 & $<0.001$ & $7.9(13.3)$ & $<0.001$ \\
\hline \multicolumn{8}{|l|}{ Operation type } \\
\hline PPG & 358 (21.6) & 17 & & 8.4 & & $4.2(10.8)$ & \\
\hline DG & $912(54.9)$ & 22 & & 8.7 & & $5.5(9.3)$ & \\
\hline TG & $390(23.5)$ & 34.1 & $<0.001$ & 12.5 & 0.016 & $8.6(13.5)$ & $<0.001$ \\
\hline \multicolumn{8}{|c|}{ Approach method } \\
\hline Laparoscopy & $998(60.1)$ & 17.7 & & 6.6 & & $4.9(9.5)$ & \\
\hline Robot & $85(5.1)$ & 19.88 & & 11.6 & & $6.9(11.9)$ & \\
\hline Open & $577(34.8)$ & 33.9 & $<0.001$ & 13.5 & $<0.001$ & $9.7(13.0)$ & $<0.001$ \\
\hline \multicolumn{8}{|c|}{ Combined resection } \\
\hline None & $1380(83.1)$ & 21.5 & & 8.2 & & $5.3(10.8)$ & \\
\hline Resected & $280(16.9)$ & 33.8 & $<0.001$ & 16.9 & $<0.001$ & $9.0(13.4)$ & $<0.001$ \\
\hline \multicolumn{8}{|c|}{ Extent of lymph node dissection } \\
\hline Under D2 & $928(56)$ & 19.9 & $<0.001$ & 7.7 & 0.007 & $5.0(9.7)$ & $<0.001$ \\
\hline D2 & $732(44)$ & 28.5 & & 11.6 & & $7.3(12)$ & \\
\hline
\end{tabular}

$P P G$ pylorus-preserving gastrectomy, $D G$ distal gastrectomy, $T G$ total gastrectomy

that the occurrence of the most severe CDC grade did not differ in comorbid patients compared to those without comorbidities. However, multiple complications tend to occur more often in comorbid patients and thus generate a higher score in the CCI.

Because there is no gold standard for measuring clinical outcomes and comparing clinical implications of different complication grades, postoperative hospital stay was considered a surrogate marker for clinical outcome. The CCI system was more strongly correlated with hospital stay than the conventional CDC grading system, and only the CCI system exhibited a meaningful correlation with a longer hospital stay (>30 days). The decrease in the postoperative stay for grade IV patients indicated that there was an oversimplification of grade III, resulting in undergrading of the complications in the CDC system. Thus, the 
Table 3 Generalized linear model for comprehensive complication index

\begin{tabular}{|c|c|c|c|c|}
\hline \multirow[t]{2}{*}{ Variables } & \multicolumn{4}{|l|}{ CCI } \\
\hline & $B$ & Standard errors & $95 \% \mathrm{CI}$ & $p$ value \\
\hline Age & 0.106 & 0.026 & 0.057 to 0.155 & 0.001 \\
\hline Sex (male) & 2.004 & 0.607 & 0.815 to 3.153 & 0.001 \\
\hline \multicolumn{5}{|l|}{ Charlson score } \\
\hline 2 & - & - & - & - \\
\hline 3 & 1.655 & 0.051 & 0.257 to 3.054 & 0.020 \\
\hline$\geq 4$ & 3.736 & 0.099 & 1.911 to 5.56 & 0.001 \\
\hline \multicolumn{5}{|l|}{ Stage } \\
\hline I & 1.957 & 1.865 & -1.701 to 5.614 & 0.294 \\
\hline II & 1.545 & 0.805 & -2.226 to 5.316 & 0.422 \\
\hline III & 2.915 & 1.549 & -0.777 to 6.608 & 0.122 \\
\hline IV & - & - & - & - \\
\hline \multicolumn{5}{|l|}{ Operation type } \\
\hline PPG & -1.621 & 0.993 & -3.569 to 0.326 & 0.103 \\
\hline DG & -2.082 & 0.768 & -3.589 to -0.575 & 0.007 \\
\hline TG & - & - & - & - \\
\hline \multicolumn{5}{|l|}{ Approach method } \\
\hline Laparoscopy & - & - & - & - \\
\hline Robot & 0.728 & 1.356 & -1.931 to 3.388 & 0.591 \\
\hline Open & 3.752 & 1.543 & -0.004 to 6.050 & 0.001 \\
\hline Combined resection & 2.011 & 0.800 & 0.489 to 3.594 & 0.011 \\
\hline \multicolumn{5}{|c|}{ Extent of lymph node dissection } \\
\hline Under D2 & 0.291 & 0.751 & -1.182 to 1.742 & 0.699 \\
\hline D2 dissection & - & & & \\
\hline
\end{tabular}

$P P G$ pylorus-preserving gastrectomy, $D G$ distal gastrectomy, $T G$ total gastrectomy, $C I$ confidence interval
CCI system offers a more precise grading system for complicated patients who require a longer hospital stay as well as for patients with severe complications compared to the CDC.

Medical cost may also be a good indicator to reflect the severity of treatment. It should represent consumed resources in a reasonable manner. However, because the cost of Korean medical services is fixed solely by the government authority and medical cost is weighted by other issues rather than medical urgency, its application might be difficult in our setting. Medication or treatment that is not reimbursed by insurance is incomparably expensive. For example, the cost for radiological versus surgical intervention shows not much difference and perhaps may be greater in some radiological interventions. Furthermore, the radiological interventions in our complicated patients required multiple sessions to diagnose, follow up, and terminate the process to fulfill its purpose. Thus, patients who received an expensive treatment option irrespective of treatment method should differ in total medical cost.

The values of the CCI range up to 100; this scale provides a wider, therefore more differentiated, range of grading for complications than the CDC. However, the CCI was not evenly distributed throughout the scale and the distribution tended to cluster in the values that represent each grade of the CDC $(8.7,20.9,26.2, \ldots$ etc. $)$, mirroring the patients with only one complication. In patients with more severe complications (CDC grade III to IV), a wider spectrum of CCI values was present. This phenomenon is explainable by the fact that more severe complications are often accompanied by additional minor complications. This wide spectrum is the result of the reflection of the overall postoperative morbidity of each patient.

For monitoring overall surgical outcomes, because the CCI system is a wide continuous scoring variable, it can be mathematically integrated and combined with the CUSUM chart. Considering both the number and severity of complications, this method provides more information about maintaining an adequate level of surgical outcome as well as prompt detection of lapses in performance than monitoring complication rates. The CUSUM-CCI model chart displays dynamic differences in time-event settings for individual surgeons and can be used to compare each of them. Moreover, the CCI CUSUM chart can function as a complication archive on past records. 

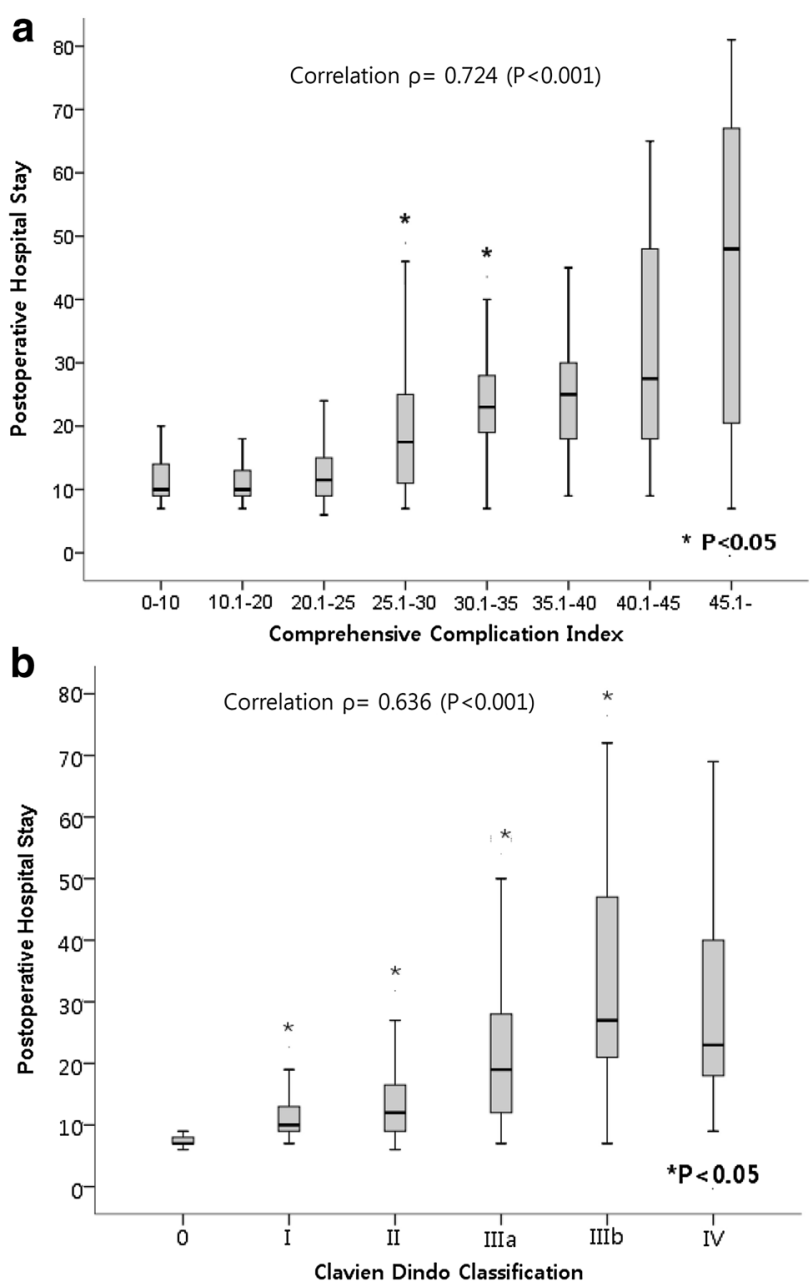

Fig. 2 Postoperative stay and complication grading methods (CCI) (a) and hospital stay. b Clavien-Dindo classification

This method provides instant review of performance for individual, institutional surgical performance and can continuously collect and monitor prospective data of complications. This activity eventually generates feedback for each surgeon to reassess surgical skills and reinforce standard procedures. This phenomenon was demonstrated by our institutional decrease in the CCI during the study period.

The usefulness of CDC should never be underestimated; the unspecific terms major and minor complication have been separated into five simple, ubiquitous, and reproducible grades. Surgical performance and short-term outcome could be measured on the occurrence rate of CDC $\geq$ IIIa. However, this oversimplification results in ignorance of true postoperative morbidity, especially in severe cases in which multiple complications or series of complications take place. The CCI displays continuous numerical figures that are easy to interpret and compare. However, the calculating process generates additional work steps, and the results are severely dependent on the quality of the complications data set.
Regarding the statistical feasibility of the CCI, linear regression can be applied to stratify risk factors and perform multivariate analysis because it is a continuous variable. However, in the case of a small volume of dataset, this might not be true, and other options should be considered.

When the CCI is analyzed in binary regression where cutoff value is required, a cutoff value of 25.2 in the CCI was shown as a good parameter to predict long hospital stay, more than 30 days $($ AUC $=0.964$; sensitivity $=0.942$, specificity $=0.918$ ) with significant difference in mean hospital days before $(12.7 \pm 5.3)$ and after $(24.4 \pm 17.4)$ this point $(p<0.001)$. However, the dichotomous transformation of CCI results in loss of data information and minimizes the CCI intended purpose of reflecting overall complication.

The strength of this study is that the CCI was tested in a prospective database and was more comprehensive than the conventional CDC. Furthermore, the advantage of using continuous variables serves as an easily applicable endpoint in complication monitoring models for surgical procedures.

Nevertheless, our study has certain limitations. First, although clinical factors were included in the analysis, our data lack factors such as the social situations of the patient in planning discharge, which may influence the timing of discharge. Second, there are some inherent shortcomings in the CCI, which is derived from the grading of the ClavienDindo classification. For instance, timely surgical intervention to correct complications is graded more severely than conservative treatment requiring a longer treatment period. Thus, the personal practice of the surgeon can affect the decision to perform interventional surgery, and therefore considerable effort is required to maintain a constant and unified treatment consensus. Calculating the $\mathrm{CCI}$, because every grade I complication is integrated and influences the index, a unified definition of the normal postoperative course is required. With the CUSUM model, the concept of maintaining good surgical quality has been introduced. When applied to different settings, the comparison model of CUSUM requires patient, surgeon, and hospital adjustment before direct comparison. The minimal requirement is sharing the same principle to treat complications, because individual surgeons could bear the risk of refusing operative treatments to patients with a high postoperative morbidity risk with the intention to keep the personal CCI score low.

In conclusion, the application of the CCI in gastrectomy patients is feasible and facilitates the evaluation of both the incidence and grade of postoperative morbidity. The associated risk factors for a high CCI included age, gender, operation type, approach method, and Charlson score. The CCI exhibits a stronger correlation with complications than 
Table 4 Multivariate analysis: predicted influence of each complication score on hospital stay

\begin{tabular}{|c|c|c|c|c|c|c|c|c|c|c|}
\hline \multirow[t]{2}{*}{ Factors } & \multicolumn{5}{|c|}{ Postoperative hospital stay } & \multicolumn{5}{|c|}{ Prolonged postoperative hospital stay $(>30)$} \\
\hline & $B$ & $\begin{array}{l}\text { Standard } \\
\text { errors }\end{array}$ & $95 \% \mathrm{CI}$ & $p$ value & $R^{2}$ & $B$ & $\begin{array}{l}\text { Standard } \\
\text { errors }\end{array}$ & $95 \% \mathrm{CI}$ & $p$ value & $R^{2}$ \\
\hline \multicolumn{11}{|c|}{ CCI and postoperative hospital stay } \\
\hline $\mathrm{CCI}$ & 0.332 & 0.008 & 0.316 to 0.348 & $<0.001$ & 0.505 & 0.704 & 0.123 & 0.457 to 0.952 & 0.001 & 0.387 \\
\hline \multicolumn{11}{|l|}{ Charlson score } \\
\hline 2 & -2.163 & 0.281 & $\begin{array}{c}-2.714 \text { to } \\
-1.613\end{array}$ & $<0.001$ & & 0.679 & 3.337 & $\begin{array}{c}-6.041 \text { to } \\
7.400\end{array}$ & 0.840 & \\
\hline 3 & -2.052 & 0.319 & $\begin{array}{c}-2.676 \text { to } \\
-1.427\end{array}$ & $<0.001$ & & 6.329 & 4.194 & $\begin{array}{c}-2.118 \text { to } \\
14.777\end{array}$ & 0.138 & \\
\hline $4 \leq$ & - & - & - & - & & & & & & \\
\hline \multicolumn{11}{|l|}{ Operation type } \\
\hline PPG & -1.152 & 0.252 & $\begin{array}{c}-1.646 \text { to } \\
-0.658\end{array}$ & $<0.001$ & & -4.900 & 4.392 & $\begin{array}{c}-13.745 \text { to } \\
3.9455\end{array}$ & 0.270 & \\
\hline DG & -0.957 & 0.208 & $\begin{array}{c}-1.366 \text { to } \\
-0.548\end{array}$ & $<0.001$ & & -1.313 & 3.264 & $\begin{array}{c}-7.886 \text { to } \\
5.260\end{array}$ & 0.689 & \\
\hline TG & - & - & - & - & & - & - & - & - & \\
\hline Constant & 10.685 & 0.312 & $\begin{array}{c}10.074 \text { to } \\
11.296\end{array}$ & $<0.001$ & & 19.291 & 6.064 & $\begin{array}{r}7.076 \text { to } \\
31.505\end{array}$ & 0.003 & \\
\hline \multicolumn{11}{|c|}{ CDC and postoperative hospital stay } \\
\hline $\begin{array}{c}\text { Clavien-Dindo } \\
\text { classification }\end{array}$ & & & & & 0.403 & & & & & 0.297 \\
\hline I & -14.289 & 1.568 & $\begin{array}{c}-17.365 \text { to } \\
-11.123\end{array}$ & $<0.001$ & & - & - & - & - & \\
\hline II & -12.045 & 1.530 & $\begin{array}{c}-15.046 \text { to } \\
-9.044\end{array}$ & $<0.001$ & & -17.220 & 6.664 & $\begin{array}{c}-30.677 \text { to } \\
-3.762\end{array}$ & 0.013 & \\
\hline IIIa & -7.704 & 1.545 & $\begin{array}{c}-10.735 \text { to } \\
4.673\end{array}$ & $<0.001$ & & -12.556 & 6.247 & $\begin{array}{c}-25.172 \text { to } \\
0.060\end{array}$ & 0.051 & \\
\hline IIIb & -1.698 & 1.838 & $\begin{array}{l}-5.304 \text { to } \\
1.908\end{array}$ & 0.356 & & 7.109 & 7.790 & $\begin{array}{c}-8.624 \text { to } \\
22.842\end{array}$ & 0.367 & \\
\hline IVa & - & - & - & & & - & - & - & - & \\
\hline \multicolumn{11}{|l|}{ Charlson score } \\
\hline 2 & -2.376 & 0.312 & $\begin{array}{c}-2.988 \text { to } \\
-1.765\end{array}$ & $<0.001$ & & 0.028 & 3.865 & $\begin{array}{c}-7.776 \text { to } \\
7.833\end{array}$ & 0.994 & \\
\hline 3 & -1.510 & 0.280 & $\begin{array}{c}-2.783 \text { to } \\
1.392\end{array}$ & $<0.001$ & & 8.309 & 4.989 & $\begin{array}{c}-1.767 \text { to } \\
18.385\end{array}$ & 0.103 & \\
\hline $4 \leq$ & - & - & - & - & & - & - & - & - & \\
\hline \multicolumn{11}{|l|}{ Operation type } \\
\hline PPG & -1.510 & 0.280 & $\begin{array}{c}-2.060 \text { to } \\
-0.961\end{array}$ & $<0.001$ & & -5.104 & 5.492 & $\begin{array}{l}-16.196 \text { to } \\
2.987\end{array}$ & 0.358 & \\
\hline DG & -1.131 & 0.232 & $\begin{array}{c}-1.586 \text { to } \\
-0.676\end{array}$ & $<0.001$ & & -1.975 & 4.296 & $\begin{array}{c}-10.652 \text { to } \\
6.701\end{array}$ & 0.648 & \\
\hline TG & - & & - & - & & - & - & - & - & \\
\hline Constant & 28.413 & 1.514 & $\begin{array}{c}25.444 \text { to } \\
31.382\end{array}$ & $<0.001$ & & 54.266 & 5.470 & $\begin{array}{c}43.219 \text { to } \\
65.312\end{array}$ & $<0.001$ & \\
\hline
\end{tabular}

$P P G$ pylorus-preserving gastrectomy, $D G$ distal gastrectomy, $T G$ total gastrectomy 
Fig. 3 Monitoring surgical complications. a Individual model: complication monitoring model with cumulative sum control (CUSUM) score for surgeon A was derived from target value of average CCI (5.4) of surgeon A in the year 2013. The final CUSUM score for year 2014 was -53.7 , which indicates a decreased CCI compared to the previous year. The CUSUM score for surgeon $\mathrm{B}$ was derived from target value of average CCI (7.4) of surgeon $B$ in the year 2013. The final score was -185.2 , which shows improved CCI score.

b Comparison model: target value was set in the mean CCI score (6.4) of cases of surgeon $A$ and surgeon B in 2013. The chart indicates constant decrease for surgeon $\mathrm{A}$; final CUSUM score indicates -414.1. Surgeon B showed gradual increase after case number 121 and the score results were 116 . c Continuous monitoring of CUSUM-CCI score of year 2013: 2014 is depicted. Target value was set in the average CCI score of the institution in year 2013 (6.1). The final CUSUM score was -212.3 , which result showed a gradual decrease throughout the observation period a
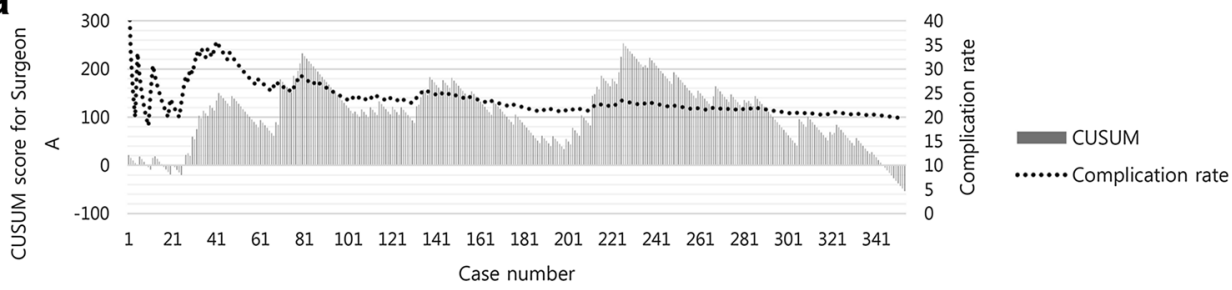

CUSUM ....... Complication rate

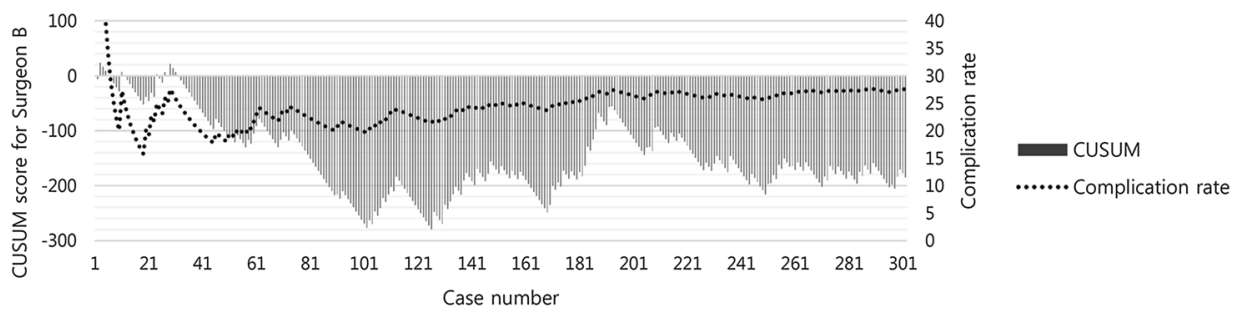

b
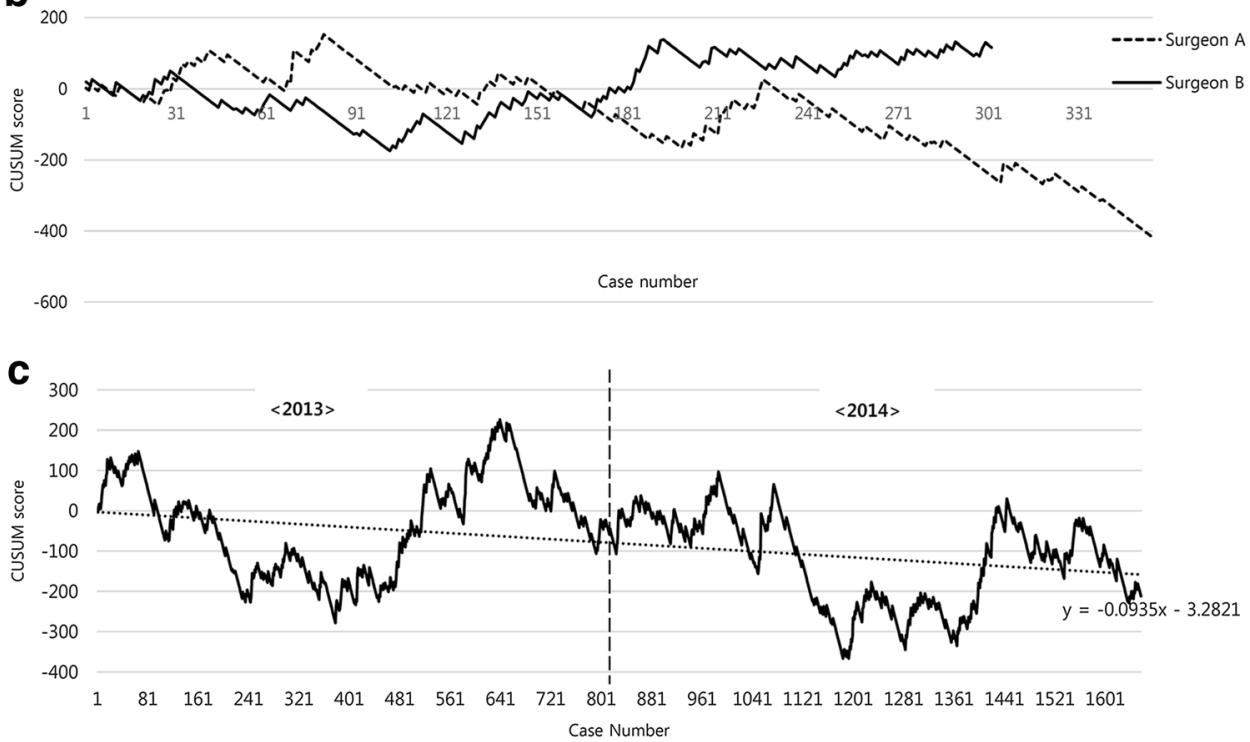

does the conventional CDC and exhibits a stronger correlation with longer hospital stays. In addition, the CCI has a definite advantage to the CDC in terms of the continual monitoring of surgical quality, including monitoring individual surgeons or presenting comparisons between surgeons.

\section{Compliance with ethical standards}

Conflict of interest The authors have no conflicts of interest to disclose. The CCI is a registered trademark owned by the University of Zurich.

Human rights statement and informed consent Our protocol was approved by the ethics committee of Seoul National University Hospital Institution Review board (IRB Approval No. 1503-095-657). The requirement for the investigator to obtain a signed consent form from each patient was waived, given the anonymous nature of the data employed. All procedures followed were in accordance with the ethical standards of the responsible committee on human experimentation (institutional and national) and with the Helsinki Declaration.

Funding This study was supported by a research grant from the Seoul National University Hospital Research Fund (0420153030).

\section{References}

1. Kim W, et al. Decreased morbidity of laparoscopic distal gastrectomy compared with open distal gastrectomy for stage I gastric cancer: short-term outcomes from a multicenter randomized controlled trial (KLASS-01). Ann Surg. 2016;263(1):28-35.

2. Sano $\mathrm{T}$, et al. Gastric cancer surgery: morbidity and mortality results from a prospective randomized controlled trial comparing D2 and extended para-aortic lymphadenectomy-Japan Clinical Oncology Group study 9501. J Clin Oncol. 2004;22(14):2767-73.

3. Lee KG, et al. Risk factors associated with complication following gastrectomy for gastric cancer: retrospective analysis of 
prospectively collected data based on the Clavien-Dindo system. J Gastrointest Surg. 2014;18(7):1269-77.

4. Kim W, et al. The impact of comorbidity on surgical outcomes in laparoscopy-assisted distal gastrectomy: a retrospective analysis of multicenter results. Ann Surg. 2008;248(5):793-9.

5. Dindo D, Demartines N, Clavien PA. Classification of surgical complications: a new proposal with evaluation in a cohort of 6336 patients and results of a survey. Ann Surg. 2004;240(2):205-13.

6. Clavien PA, et al. The Clavien-Dindo classification of surgical complications: five-year experience. Ann Surg. 2009;250(2):187-96.

7. Bruce J, et al. Systematic review of the definition and measurement of anastomotic leak after gastrointestinal surgery. Br J Surg. 2001;88(9):1157-68.

8. Yoon PD, Chalasani V, Woo HH. Use of Clavien-Dindo classification in reporting and grading complications after urological surgical procedures: analysis of 2010-2012. J Urol. 2013;190(4):1271-4.

9. Wechter ME, et al. Complications in robotic-assisted gynecologic surgery according to case type: a 6-year retrospective cohort study using Clavien-Dindo classification. J Minim Invasive Gynecol. 2014;21(5):844-50.

10. Sun LP, et al. Correlation between an insertion-deletion polymorphism in the pepsinogen $\mathrm{C}$ gene and gastric cancer as well as its precursors. Zhonghua Yi Xue Za Zhi. 2006;86(43):3059-63.

11. Slankamenac $K$, et al. The comprehensive complication index: a novel and more sensitive endpoint for assessing outcome and reducing sample size in randomized controlled trials. Ann Surg. 2014;260(5):757-62 (discussion 762-763).

12. Slankamenac $\mathrm{K}$, et al. The comprehensive complication index: a novel continuous scale to measure surgical morbidity. Ann Surg. 2013;258(1):1-7.

13. Washington K. 7th edition of the AJCC cancer staging manual: stomach. Ann Surg Oncol. 2010;17(12):3077-9.

14. Japanese Gastric Cancer Association. Japanese gastric cancer treatment guidelines 2010 (ver. 3). Gastric Cancer. 2011;14(2):113-23.
15. Charlson ME, et al. A new method of classifying prognostic comorbidity in longitudinal studies: development and validation. J Chronic Dis. 1987;40(5):373-83.

16. Huang $\mathrm{CM}$, et al. A scoring system to predict the risk of postoperative complications after laparoscopic gastrectomy for gastric cancer based on a large-scale retrospective study. Medicine (Baltim). 2015;94(17):e812.

17. Slankamenac K, et al. Perception of surgical complications among patients, nurses and physicians: a prospective cross-sectional survey. Patient Saf Surg. 2011;5(1):30.

18. Connolly TM, Watters DA. Monitoring performance in thyroidectomy: cumulative sum analysis of outcomes. Thyroid. 2010;20(4):407-12.

19. Mittlbock M, Heinzl H. A note on R2 measures for Poisson and logistic regression models when both models are applicable. J Clin Epidemiol. 2001;54(1):99-103.

20. Borchert DH, et al. Comprehensive complication index for NOTES procedures: results from a randomized controlled trial and comparison to published NOTES complication data. Surg Endosc. 2015;29(10):2928-33.

21. Slaman AE, et al. A quantified scoring system for postoperative complication severity compared to the Clavien-Dindo classification. Dig Surg. 2015;32(5):361-6.

22. Laor A, et al. The Charlson comorbidity index (CCI) as a mortality predictor after surgery in elderly patients. Am Surg. 2016;82(1):22-7.

23. Crooks CJ, West J, Card TR. A comparison of the recording of comorbidity in primary and secondary care by using the Charlson index to predict short-term and long-term survival in a routine linked data cohort. BMJ Open. 2015;5(6):e007974.

24. Sarfati D, et al. Cancer-specific administrative data-based comorbidity indices provided valid alternative to Charlson and National Cancer Institute Indices. J Clin Epidemiol. 2014;67(5):586-95.

25. Lieffers JR, et al. A comparison of Charlson and Elixhauser comorbidity measures to predict colorectal cancer survival using administrative health data. Cancer (Phila). 2011;117(9):1957-65. 